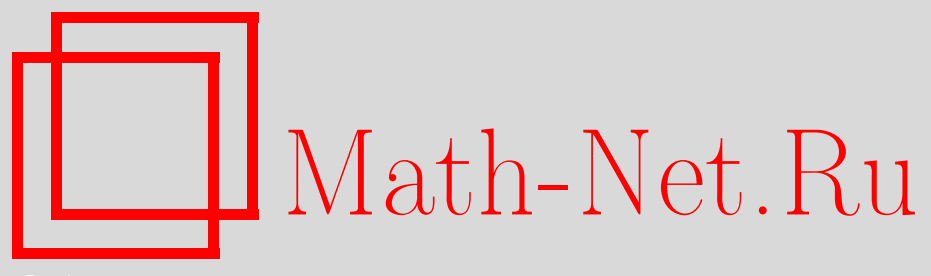

В. П. Маслов, Сравнение новой теории нуклеации с экспериментальными данными, Матем. заметки, 2008, том 84, выпуск 1, 149-152

DOI: https://doi.org/10.4213/mzm5199

Использование Общероссийского математического портала Math-Net.Ru подразумевает, что вы прочитали и согласны с пользовательским соглашением http://www . mathnet.ru/rus/agreement

Параметры загрузки:

IP: 52.23 .180 .231

26 апреля 2023 г., 18:21:47

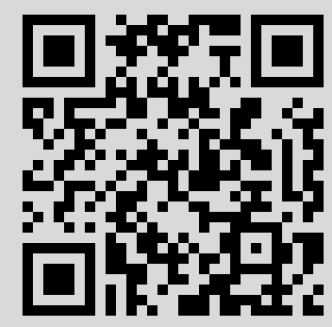




\section{Сравнение новой теории нуклеации с экспериментальными данными}

\section{В. П. Маслов}

В задаче термодинамики есть большой параметр - число частиц. Кроме того, имеется другой большой параметр объем $V$. Мы рассматриваем удельный объем $V_{\text {уд }}=V / N$. Объем $V$ имеет 3-мерную размерность.

Термодинамическая наука также рассматривает 2-мерную поверхность с большим параметром - числом частиц $N^{2 / 3}$ и удельную поверхность $S / N^{2 / 3}$, где $S$ - площадь поверхности. Очевидно, что между этими двумя целыми размерностями есть и другие нецелые размерности, которые получаются, когда удельный объем стремится к нулю.

Иначе говоря, когда объем $V$ есть утолщенная поверхность, толщина которой $D \approx$ $N^{\alpha-1 / 3}$ при $\alpha<1 / 3$ стремится к нулю при $N \rightarrow \infty$, при $\alpha=0$ получается поверхность $S$. Величина $\alpha$ определяет промежуточную размерность между размерностью 3 и 2 . Если речь идет о газе, то реально она может быть определена нанопорами в веществе и их объемом.

Максимальное число частиц в перенасыщенном газе (паре) в 3-мерном объеме определяется из распределения типа Бозе-Эйнштейна. Для 2-мерного случая поверхности оно равно бесконечности.

Однако для промежуточной размерности, определенной нанопорами, оно конечно и стремится к бесконечности при размерности, стремящейся к 2. Если газ перенасыщенный при фиксированной достаточно низкой температуре, то его минимальная сжимаемость $Z=P V / k T N \cong 0.53, P$ - давление, $T$ - температура, $k$ - константа Больцмана. Однако при этом может образовываться жидкость с нанопузырями, и размерность может резко упасть при увеличении давления, а следовательно, может упасть сжимаемость. (Сжимаемость 2-мерной задачи идеального газа с распределением типа Бозе-Эйнштейна равна нулю.)

Рассмотрим вначале графики на рис. 1 и 2 (для аргона; давление приведено в паскалях, температура в кельвинах).

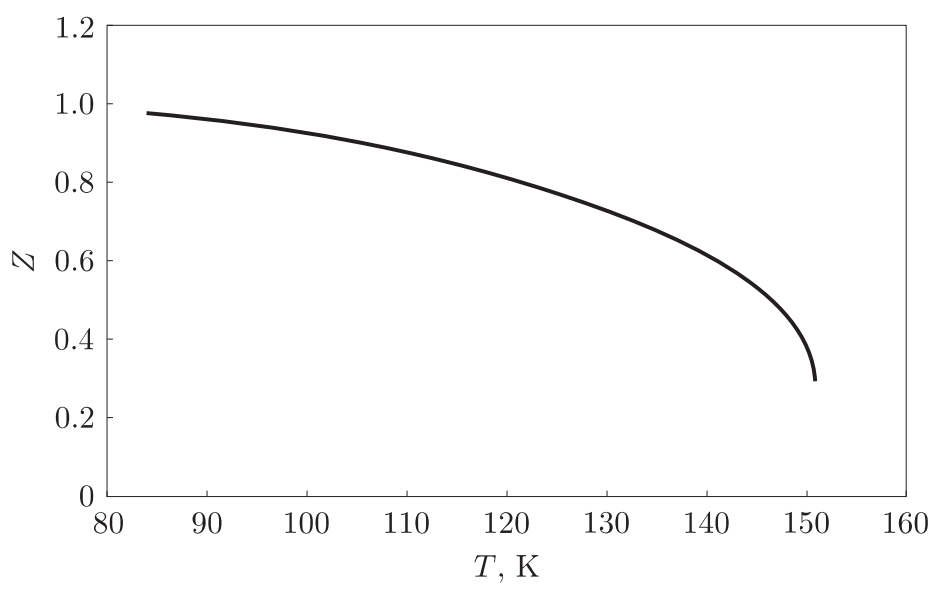

Рис. 1

Если пар насыщенный, то при низкой температуре, как правило, число кластеров (димеров, тримеров) велико. Это уменьшает общее число частиц в объеме и увеличивает

Автор предоставил более развернутый вариант статьи для английского перевода.

(C) В. П. МАслов, 2008 


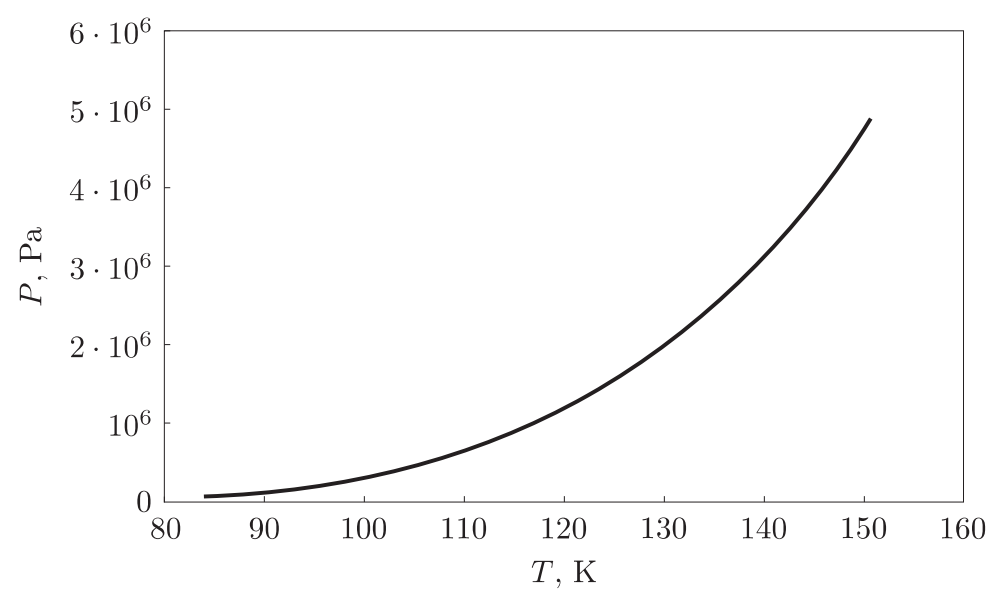

Рис. 2

химпотенциал, а значит, увеличивается сжимаемость. При увеличении температуры число кластеров уменьшается, и при некоторой температуре димеров становится меньше $7 \%$ (критерий Кало). Тогда сжимаемость может упасть до 0.53.

Но в силу того, что насыщенный газ находится в равновесии с жидкостью, которая содержит нанопузырьки, размерность после этого может достаточно резко упасть и сжимаемость (для аргона, например) уменьшится до 0.25 .

Таким образом, образование наноструктур в другой фазе (жидкости) играет существенную роль, как и образование кластеров в газе. Будем в дальнейшем называть такое образование наножидкостью размерности $2<q<3$, отвечающей величине сжимаемости.

Следовательно, изменение размерности, которое происходит почти скачкообразно (см. графики на рис. 1,2 и 3), может вызвать возникновение ударной волны в жидкости. Это наблюдается, в частности, на атомных станциях, когда жидкость, протекая между твелами (тепловыделяющими элементами), превращается в пар, вращающий лопасти, размерность меняется периодически, и ударные волны разрушают конструкцию.

Теперь перейдем к случаю постоянной температуры (рис. $3 ; T_{r}=T / T_{c}$, и $P_{r}=P / P_{c}-$ приведенные температура и давление соответственно [1]).

Рассмотрим предварительно 3-мерную задачу

$$
\Delta U_{n}+\lambda_{n} U_{n}=0
$$

в объеме $V$ с условиями Дирихле на $\delta V$. В силу теоремы Куранта (правило Вейля) число собственных значений задачи при

$$
\lambda_{n} \leqslant E
$$

где $E \rightarrow \infty$, с учетом кратностей асимптотически приближается к фазовому объему $\sqrt{E^{3}} V$. Можно однако фиксировать $E$, а $V$ стремить к бесконечности. Тогда можно считать, что вместо $E$ к бесконечности стремится $V^{2 / 3}$. А значит, во всех формулах типа Бозе-Эйнштейна в [2], [3] полагать $b^{-1} \simeq V^{2 / d} / k T$, где $T$ - температура, $k$ - константа Больцмана, $d$ - размерность.

Чтобы число $b$ в [2], [3] было безразмерно, введем еще $a$ - эффективный радиус молекулы газа (см. ниже). Тогда

$$
b=\left(\frac{V}{a^{3}}\right)^{-2 / d} \frac{E_{0}}{k T},
$$

где $E_{0}$ определено в [2], [3]. 


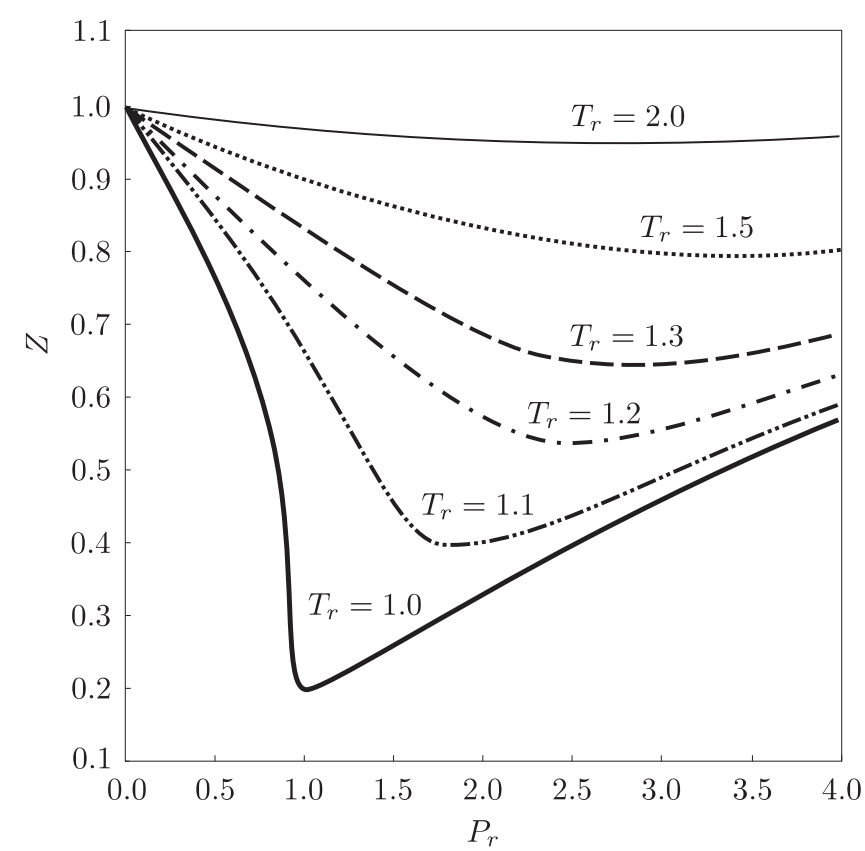

Рис. 3

Предположим, что число частиц $N$ задано. Тогда давление $P$ в 3 -мерном случае равно

$$
P=\frac{1}{N} \int \frac{p^{2} / 2 m}{\exp \left\{\frac{a^{2}}{V^{2 / 3}} \frac{E_{0}}{k T} \frac{p^{2}}{2 m}-\frac{\mu}{k T}\right\}-1} d p_{1} d p_{2} d p_{3},
$$

$p^{2}=p_{1}^{2}+p_{2}^{2}+p_{3}^{2}, \mu$-химпотенциал, $\mu \leqslant 0$. Если $N=N_{0}$ - число частиц перенасыщенного газа $(\mu=0)$, то $P=$ const $\cdot V^{-5 / 3}$.

При увеличении объема отрицательный химпотенциал $\mu$ уменьшается (увеличивается по абсолютной величине). Значение химпотенциала определяется при фиксированной температуре и фиксированном числе частиц $N$ из формулы

$$
\int \frac{\sqrt{\xi} d \xi}{\exp \{\xi-\mu / k T\}-1}=\operatorname{Li}_{3 / 2} \exp \left\{\frac{\mu}{k T}\right\}=\frac{N a^{3} E_{0}^{3 / 2}}{V(k T)^{3 / 2}} \text { const. }
$$

Однако если объем сильно уменьшается, то газ перестает быть идеальным, и нужно учесть одетый потенциал.

Для этого рассмотрим притяжение вида потенциала Ленард-Джонса

$$
\Phi(r)=4 \varepsilon\left\{\left(\frac{a}{r}\right)^{12}-\left(\frac{a}{r}\right)^{6}\right\}, \quad r \geqslant a, \quad \Phi(r)=0, \quad r \leqslant a,
$$

где $\varepsilon$ и $a$ - постоянные, имеющие размерность энергии и длины соответственно;

$$
r^{2}=\left(x_{i}-x_{j}\right)^{2}+\left(y_{i}-y_{j}\right)^{2}+\left(z_{i}-z_{j}\right)^{2}, \quad i \neq j,
$$

$x_{i}, y_{i}, z_{i}$ - координаты $i$-й частицы. Длина $a$ есть эффективный поперечник частицы; при $r=a$ имеем $\Phi(a)=0$. Величина $\varepsilon$ определяет глубину потенциальной ямы $\Phi(r)$ и, следовательно, характеризует интенсивность межмолекулярных сил. 
Напишем уравнение для одетого потенциала [3]

$$
U(x)=\int \Phi(x-\xi) \operatorname{Li}_{3 / 2}\left(e^{-b U(\xi)}\right) d \xi .
$$

Здесь $\Phi(x-\xi)$ - потенциал (5), a $\mathrm{Li}_{3 / 2}$ - полилогарифм. Его можно переписать, поставив вместо ядра интегрального оператора псевдодифференциальный оператор $\widetilde{\Phi}(i a \partial / \partial x)$, где $\widetilde{\Phi}$ - преобразование Фурье от потенциала взаимодействия.

В первом приближении при $a \rightarrow 0$ из соотношения

$$
C=\widetilde{\Phi}(0) \operatorname{Li}_{3 / 2}\left(e^{-b C}\right), \quad \widetilde{\Phi}(0)=\int_{a}^{\infty} \Phi(r) d x d y d z,
$$

где $r^{2}=x^{2}+y^{2}+z^{2}$, может быть определена константа $C$.

При $a^{3} / V \ll 1$ ( $V$ - объем) можно полагать, что интеграл (7) берется по отрицательной части потенциала (5). Если $a^{3} N \ll V$ (где $N$ - число частиц), то взаимодействием можно пренебречь.

Если его учесть, но считать достаточно малым, то в соотношении (3) возникает дополнительная добавка к химпотенциалу, равная $-C$.

Если положить

$$
V(\lambda)=\int_{H(q, p) \leqslant \lambda} d q d p
$$

в терхмерном пространстве, где $H(p, q)$ - гамильтониан, и $V^{\prime}(\lambda)$ - достаточно гладкая функция, то средняя энергия перенасыщенного газа по-прежнему будет пропорциональна $1 / b$.

Если же предположить, что взаимодействие включается лишь при энергии меньшей, чем глубина $\varepsilon$ потенциальной ямы (5), мы можем воспользоваться приведенной выше формулой для давления с учетом этого "скачка". При определенных соотношениях на $C$, $\mu(V, T), T$ и $\varepsilon$ возникнут два горба типа зависимости давления от объема в формуле Ван-дер-Ваальса, которая в равновесном состоянии определяет грубый фазовый переход из газообразного состояния в жидкое.

Выражаю глубокую благодарность академику А. К. Реброву и профессору Б. В. Егорову за предоставление экспериментальных данных.

\section{СПИСОК ЦИТИРОВАННОЙ ЛИТЕРАТУРЫ}

[1] Compressibility factor (gases), http://en.citizendium.org/wiki/Compressibility_factor. [2] В.П. Маслов, Матем. заметки, 83:5 (2008), 787-791. [3] В.П. Маслов, Матем. заметки, 83:6 (2008), 864-879.

\section{В. П. Маслов}

